\title{
First principles analysis of hydrogen chemisorption on Pd-Re alloyed overlayers and
} alloyed surfaces

\author{
Pallassana, Venkataraman; Neurock, Matthew; Hansen, Lars Bruno; Nørskov, Jens Kehlet
}

Published in:

Journal of Chemical Physics

Link to article, DOI:

$10.1063 / 1.481119$

Publication date:

2000

Document Version

Publisher's PDF, also known as Version of record

Link back to DTU Orbit

Citation (APA):

Pallassana, V., Neurock, M., Hansen, L. B., \& Nørskov, J. K. (2000). First principles analysis of hydrogen

chemisorption on Pd-Re alloyed overlayers and alloyed surfaces. Journal of Chemical Physics, 112(12), 54355439. https://doi.org/10.1063/1.481119

\section{General rights}

Copyright and moral rights for the publications made accessible in the public portal are retained by the authors and/or other copyright owners and it is a condition of accessing publications that users recognise and abide by the legal requirements associated with these rights.

- Users may download and print one copy of any publication from the public portal for the purpose of private study or research.

- You may not further distribute the material or use it for any profit-making activity or commercial gain

- You may freely distribute the URL identifying the publication in the public portal 


\title{
First principles analysis of hydrogen chemisorption on Pd-Re alloyed overlayers and alloyed surfaces
}

\author{
Venkataraman Pallassana and Matthew Neurock \\ Department of Chemical Engineering, University of Virginia, Charlottesville, Virginia 22903 \\ Lars B. Hansen and Jens K. Nørskov \\ Center for Atomic-scale Materials Physics (CAMP), Department of Physics, \\ Technical University of Denmark, DK-2800 Lyngby, Denmark
}

(Received 4 October 1999; accepted 7 January 2000)

\begin{abstract}
Gradient corrected periodic density functional theory (DFT-GGA) slab calculations were used to examine the chemisorption of atomic hydrogen on various Pd-Re alloyed overlayers and uniformly alloyed surfaces. Adsorption was examined at 33\% surface coverage, where atomic hydrogen preferred the three-fold fcc sites. The binding energy of atomic hydrogen is observed to vary by as much as $0.7 \mathrm{eV}$ due to $\mathrm{Pd}-\mathrm{Re}$ interactions. The computed adsorption energies were found to be between $-2.35 \mathrm{eV}$ [for monolayer Pd-on-Re, i.e., $\left.\mathrm{Pd}_{\mathrm{ML}} / \mathrm{Re}(0001)\right]$ and $-3.05 \mathrm{eV}$ [for $\left.\mathrm{Pd}_{33} \operatorname{Re}_{66} / \mathrm{Pd}(111)\right]$. A $d$-band weighting scheme was developed to extend the Hammer-Nørskov surface reactivity model [Surf. Sci. 343, 211 (1995)] to the analysis of bimetallic Pd-Re alloyed systems. The hydrogen chemisorption energies are correlated linearly to the surface $d$-band center, which is weighted appropriately by the $d$-band coupling matrix elements for Pd and Re. The farther the weighted $d$-band center is shifted below the Fermi energy, the weaker is the interaction of atomic hydrogen with the alloyed Pd-Re surface. (C) 2000 American Institute of Physics.
\end{abstract}

[S0021-9606(00)70809-9]

\section{INTRODUCTION}

Much of the petrochemical industry practices hydrogenation of unsaturated hydrocarbon feeds over supported bimetallic particles. ${ }^{1}$ The synergy between the two metals often leads to catalytic reactivity that is significantly different as compared to each of the monometallic components. An improved understanding of how bimetallic metal-metal interaction influences surface reactivity will likely provide insights into designing surface alloys with specific catalytic properties. Over the past decade, first-principles density functional (DFT) calculations ${ }^{2-5}$ and UHV surface-science studies $^{6-13}$ have helped to elucidate the chemical reactivity of well-defined pseudomorphic overlayers and uniformly alloyed surfaces. Simplified models have been proposed to correlate changes in chemical reactivity to electronic perturbations in these idealized surfaces. Goodman and coworkers, for instance, showed how changes in the chemisorption energy of $\mathrm{CO}$ on pseudomorphic overlayers, correlate with measured XPS shifts in the core-level binding energies for the surface metal. ${ }^{6,7,14,15}$ Using frontier orbital theory ${ }^{16,17}$ and the Newns-Anderson chemisorption model, ${ }^{18}$ Hammer and Nørskov developed a simplified chemisorption and reactivity model, ${ }^{3,19-21}$ which demonstrated that the metal-adsorbate bond strength is closely related to the surface metal $d$-band structure. The core-level shifts measured by Goodman and co-workers were shown to be commensurate with changes in the valence $d$-band center. ${ }^{3}$ Recent results have verified the effectiveness of this simple model in the analysis of chemisorption of adsorbates such as $\mathrm{CO},{ }^{3,22}$ $\mathrm{H}_{2},{ }^{21,23-25} \mathrm{NO},{ }^{26,27}$ ethylene $^{28}$ and maleic anhydride ${ }^{5}$ on monometallic and pseudomorphic overlayer surfaces. The simple two-level interaction model, ${ }^{3,5,20,23,24}$ which treats orbital overlap and Pauli repulsion, was also found to provide excellent correlation between activation barriers, for bondbreaking and coupling reactions on pseudomorphic overlayer surfaces, and shifts in the position of the surface $d$-band center. $^{4,28}$

The original model proposed by Hammer and Nørskov ${ }^{19}$ has been predominantly used to examine the interaction of adsorbates, such as $\mathrm{CO}$ and atomic hydrogen, with a single type of metal atom on monometallic or pseudomorphic overlayer surfaces. The changes in the reactivity were found to correlate well with the $d$-band center of the metal to which the adsorbate was bound. For a surface alloy, the situation may conceivably be more complicated, where adsorption in a multifold site may result in the adsorbate forming bonds with more than one type of metal atom. For example, species such as atomic carbon or hydrogen are most strongly bound to three-fold or four-fold hollow sites on a metal surface. On an alloyed surface, it is likely that the atomic adsorbate may form bonds concurrently with two or more types of metal atoms. In this paper, we demonstrate that the $d$-band center model of Ref. 21 may also be extended, in some cases, to describe such adsorption scenarios. To illustrate this point we consider the chemisorption of atomic hydrogen in the threefold fcc sites on various Pd-Re alloyed surfaces.

\section{COMPUTATIONAL METHODS}

DFT-GGA periodic slab calculations were performed to examine the chemisorption of atomic hydrogen for a $33 \%$ surface coverage of the fcc hollow sites. The $(\sqrt{ } 3$ $\times \sqrt{ } 3) \mathrm{R} 30^{\circ}$ unit cell was used to model the periodic adsorp- 
(a)

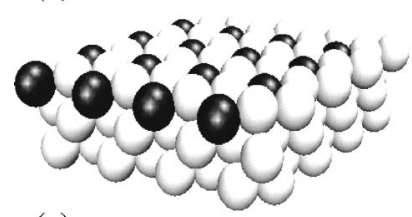

(c)

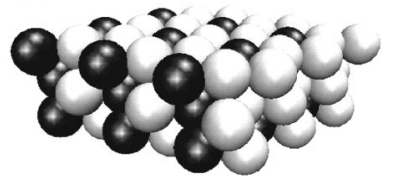

(e)

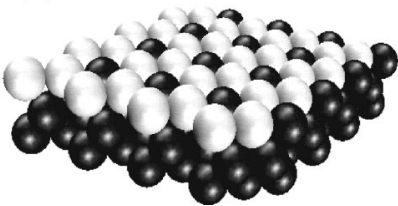

(b)

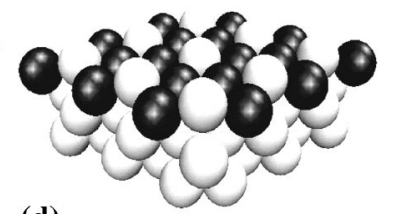

(d)

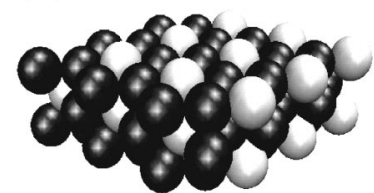

(f)

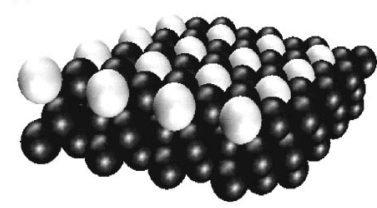

FIG. 1. Pd-Re alloyed surfaces examined in this paper for the chemisorption of atomic hydrogen. (a) 66:33 Pd:Re alloyed monolayer on Pd(111); (b) 33:66 Pd:Re alloyed monolayer on Pd(111); (c) 66:33 Pd:Re alloy (fcc); (d) 33:66 Pd:Re alloy (hcp); (e) 66:33 Pd:Re alloyed monolayer on $\operatorname{Re}(0001)$; and (f) 33:66 Pd:Re alloyed monolayer on $\operatorname{Re}(0001)$.

tion of hydrogen for 1/3 ML coverage of the surface. The fcc hollow site was determined to be the most favorable adsorption site for hydrogen chemisorption on the monometallic $\operatorname{Pd}(111)$ and $\operatorname{Re}(0001)$ surfaces and was therefore chosen for this study. ${ }^{25}$ The Kohn-Sham equations were solved selfconsistently using the Perdew-Wang 91 (PW91) 29,30 exchange-correlation functional. The inner-shell electrons of all the atoms were described by scalar-relativistically corrected, norm-conserving pseudopotentials. ${ }^{31,32}$ The maximum kinetic energy of the plane wave basis was 40 Rydberg. For a $(\sqrt{ } 3 \times \sqrt{ } 3)$ unit cell 18 Chadi-Cohen ${ }^{33} k$ points were found to be adequate for sampling the Brillouin zone and were used in all calculations. Details of the calculation procedure are available in Ref. 25.

\section{RESULTS AND DISCUSSIONS}

The bimetallic Pd-Re surfaces that were examined for hydrogen chemisorption are shown in Fig. 1. In a previous study, we examined the effect of the number of metal layers on the hydrogen chemisorption energy and found negligible changes in the adsorption energy by increasing the number of metal layers beyond three. ${ }^{25}$ Three metal layer slabs are therefore used throughout this study. Slabs (a), (b), (e), and (f) in Fig. 1, represent uniformly alloyed Pd-Re monolayers, pseudomorphically placed on a monometallic $\mathrm{Pd}(111)$ or $\operatorname{Re}(0001)$ substrate. The slab surfaces (c) and (d) are uniformly alloyed Pd-Re surfaces containing a 33:66 and 66:33 ratio of $\mathrm{Re}: \mathrm{Pd}$, respectively. Since the bulk crystal structure for $\mathrm{Re}$ is hcp, the alloyed surface containing more than $50 \%$ Re was modeled using an hcp pattern. Analogously, an fcc pattern was used for the Pd-rich alloyed surface. At the initial structure, the surfaces were chosen to have the lattice parameter of the metal with the larger atomic concentration in the bulk. Since the optimized interatomic distances in monometallic $\operatorname{Pd}(111)(2.75 \AA)$ and $\operatorname{Re}(0001)(2.76 \AA)$ are

TABLE I. Structural and energetics parameters for hydrogen chemisorption on $\operatorname{Pd}(111), \operatorname{Re}(0001)$, and $\mathrm{Pd}-\mathrm{Re}$ bimetallic surfaces. The binding energies reported are for $33 \%$ coverage of the fcc hollow sites, corresponding to a $\sqrt{3} \times \sqrt{3}$ unit cell.

\begin{tabular}{|c|c|c|c|}
\hline \multirow[b]{2}{*}{ Surface } & \multicolumn{2}{|c|}{ Metal-hydrogen bond distance $(\AA)$} & \multirow{2}{*}{$\begin{array}{c}\text { Hydrogen } \\
\text { binding } \\
\text { energy }(\mathrm{eV})\end{array}$} \\
\hline & $\mathrm{Pd}-\mathrm{H}$ & $\mathrm{Re}-\mathrm{H}$ & \\
\hline $\operatorname{Pd}(111)$ & 1.80 & & -2.77 \\
\hline $\operatorname{Re}(0001)$ & & 1.91 & -2.98 \\
\hline $\mathrm{Pd}_{\mathrm{ML}} / \operatorname{Re}(0001)$ & 1.85 & & -2.35 \\
\hline $\operatorname{Re}_{\mathrm{ML}} / \operatorname{Pd}(111)$ & & 1.94 & -2.94 \\
\hline $\mathrm{Pd}_{66} \mathrm{Re}_{33} / \mathrm{Pd}(111)$ & 1.94 & 1.84 & -2.73 \\
\hline $\mathrm{Pd}_{33} \operatorname{Re}_{66} / \mathrm{Pd}(111)$ & 2.06 & 1.87 & -3.05 \\
\hline $\mathrm{Pd}_{66} \mathrm{Re}_{33} / \operatorname{Re}(0001)$ & 1.85 & 1.91 & -2.48 \\
\hline $\mathrm{Pd}_{33} \operatorname{Re}_{66} / \operatorname{Re}(0001)$ & 1.99 & 1.86 & -2.78 \\
\hline $\mathrm{Pd}_{66} \mathrm{Re}_{33}$ alloy & 1.86 & 1.80 & -2.80 \\
\hline $\mathrm{Pd}_{33} \mathrm{Re}_{66}$ alloy & 2.02 & 1.81 & -2.90 \\
\hline
\end{tabular}

very similar, the choice of the lattice parameter at the starting geometry should not introduce significant strain in the lattice. During the geometry optimization scheme, the adsorbate and the first two metal layers of the slab were completely relaxed. Throughout the following discussion, overlayer or surface layer refers to the top metal layer to which hydrogen is bound. Substrate refers to the second and third metal layers.

Table I shows the DFT-GGA optimized metalhydrogen distance for hydrogen chemisorption on monometallic Pd(111), Re(0001), and the various alloyed Pd-Re surfaces. It is observed that when hydrogen is bonded to both $\mathrm{Pd}$ and $\mathrm{Re}$, the $\mathrm{Pd}-\mathrm{H}$ bond distances are significantly longer than that on $\operatorname{Pd}(111)(1.8 \AA)$. The $\mathrm{Re}-\mathrm{H}$ distances in these cases are slightly shorter than they are on $\operatorname{Re}(0001)(1.91 \AA)$, suggesting that atomic hydrogen forms stronger bonds with the surface Re atoms as compared to the surface Pd atoms. This is especially evident for the surfaces containing $66 \%$ $\mathrm{Re}$, where the $\mathrm{Pd}-\mathrm{H}$ distance $(2.0 \AA)$ is about $0.2 \AA$ longer than that on $\operatorname{Pd}(111)$, and hydrogen is almost bridge bound over the Re atoms. The surface structure in this case can to some extent be considered as Re dimers embedded in a $\mathrm{Pd}(111)$ surface, with atomic hydrogen almost bridge bound to the dimer.

Table I summarizes the DFT-GGA computed binding energies (BE) for atomic hydrogen on the monometallic $\mathrm{Pd}(111), \operatorname{Re}(0001)$, and bimetallic $\mathrm{Pd}-\mathrm{Re}$ surfaces examined in this study. Note that the BE for $(\sqrt{3} \times \sqrt{3})$ adsorption of atomic hydrogen reported here are about $0.1-0.15 \mathrm{eV}$ stronger than the BE reported earlier for $100 \%$ surface coverage. ${ }^{25}$ The strengthening of the adsorption energy with decrease in surface coverage is primarily due to the reduction in throughspace repulsive interactions and is discussed in detail in Ref. 25 . The resulting DFT-computed adsorption energies for hydrogen on monometallic $\operatorname{Pd}(111)(-2.77 \mathrm{eV})$ and $\operatorname{Re}(0001)$ $(-2.98 \mathrm{eV})$ are in reasonably good agreement with previously reported theoretical calculations and experimental measurements. ${ }^{34-37}$ Calculations suggest that the interaction of atomic hydrogen with the $\mathrm{Pd}_{\mathrm{ML}} / \mathrm{Re}(0001)$ and $\mathrm{Pd}_{66} \mathrm{Re}_{33} / \operatorname{Re}(0001)$ surfaces are relatively weak with binding energies of $-2.35 \mathrm{eV}$ and $-2.48 \mathrm{eV}$, respectively. These 
TABLE II. DFT-GGA computed surface electronic properties of bare $\operatorname{Pd}(111), \operatorname{Re}(0001)$, and bimetallic Pd-Re surfaces.

\begin{tabular}{|c|c|c|c|c|c|c|}
\hline \multirow[b]{2}{*}{ Surface } & \multirow{2}{*}{$\begin{array}{c}\text { Work } \\
\text { function } \\
\mathrm{eV}\end{array}$} & \multicolumn{3}{|c|}{$\begin{array}{c}d \text {-band center relative to } E_{f} \\
(\mathrm{eV})\end{array}$} & \multicolumn{2}{|c|}{$\begin{array}{l}d \text {-band filling } \\
\text { (fraction) }\end{array}$} \\
\hline & & $\mathrm{Pd}$ & $\operatorname{Re}$ & Weighted & $\mathrm{Pd}$ & $\operatorname{Re}$ \\
\hline $\operatorname{Pd}(111)^{\mathrm{a}}$ & 5.42 & -1.98 & & -1.98 & 0.9613 & \\
\hline $\operatorname{Re}(0001)^{\mathrm{a}}$ & 5.07 & & -1.16 & -1.16 & & 0.6998 \\
\hline $\mathrm{Pd}_{\mathrm{ML}} / \operatorname{Re}(0001)^{\mathrm{a}}$ & 5.23 & -2.70 & & -2.70 & 0.9608 & \\
\hline $\operatorname{Re}_{\mathrm{ML}} / \mathrm{Pd}(111)^{\mathrm{a}}$ & 5.65 & & -1.41 & -1.41 & & 0.6789 \\
\hline $\mathrm{Pd}_{66} \operatorname{Re}_{33} / \mathrm{Pd}(111)$ & 5.46 & -2.57 & -1.69 & -2.11 & 0.9507 & 0.7076 \\
\hline $\mathrm{Pd}_{33} \operatorname{Re}_{66} / \mathrm{Pd}(111)$ & 5.66 & -2.42 & -1.34 & -1.54 & 0.9512 & 0.7120 \\
\hline $\mathrm{Pd}_{66} \operatorname{Re}_{33} / \operatorname{Re}(0001)$ & 5.09 & -2.97 & -1.69 & -2.30 & 0.9499 & 0.7219 \\
\hline $\mathrm{Pd}_{33} \operatorname{Re}_{66} / \operatorname{Re}(0001)$ & 5.17 & -3.16 & -1.49 & -1.80 & 0.9499 & 0.7329 \\
\hline $\mathrm{Pd}_{66} \mathrm{Re}_{33}$ alloy & 5.31 & -2.73 & -1.59 & -2.13 & 0.9497 & 0.7178 \\
\hline $\mathrm{Pd}_{33} \operatorname{Re}_{66}$ alloy & 5.34 & -2.97 & -1.49 & -1.76 & 0.9501 & 0.7245 \\
\hline
\end{tabular}

${ }^{\mathrm{a}}$ From Ref. 25 [V. Pallassana et al., Phys. Rev B 60, 6146 (1999)].

binding energies are significantly weaker than those for hydrogen on the monometallic $\operatorname{Pd}(111)(-2.77 \mathrm{eV})$ and $\operatorname{Re}(0001)(-2.98 \mathrm{eV})$ surfaces. This indicates that the $\operatorname{Re}(0001)$ substrate significantly weakens the interaction of atomic hydrogen with the surface metal atoms. Although there is no reported experimental estimate for the binding energy of hydrogen on the $\mathrm{Pd}_{\mathrm{ML}} / \operatorname{Re}(0001)$ surface, it is known experimentally that adsorbates such as $\mathrm{CO}$ bind weaker to this pseudomorphic overlayer as compared to $\operatorname{Pd}(111)$ and $\operatorname{Re}(0001) .{ }^{6,14,15}$ Our results are consistent with this experimental observation.

Table I indicates that the hydrogen-surface bond strength for most of the bimetallic Pd-Re surfaces are between $-2.73 \mathrm{eV}$ and $-2.94 \mathrm{eV}$, which is intermediate to metalhydrogen surface bond strengths for monometallic $\operatorname{Pd}(111)$ and $\operatorname{Re}(0001)$ surfaces. The binding of atomic hydrogen on the $\mathrm{Pd}_{33} \operatorname{Re}_{66} / \mathrm{Pd}(111)(-3.05 \mathrm{eV})$ surface, however, is slightly stronger than on the $\mathrm{Pd}-\mathrm{Re}$ alloyed and monometallic $\operatorname{Pd}(111), \operatorname{Re}(0001)$ surfaces. An important observation from this study is that the variation in the hydrogen binding energy over the various $\mathrm{Pd}-\mathrm{Re}$ alloyed surfaces examined here is about $0.7 \mathrm{eV}$. This implies that the energy for dissociative adsorption of hydrogen can vary by as much as 1.4 $\mathrm{eV}$, depending on the bimetallic $\mathrm{Pd}-\mathrm{Re}$ ensemble to which hydrogen is bound. The second important conclusion from Table I is that bimetallic Pd-Re surfaces can exhibit metalhydrogen bond strengths that are significantly weaker, or sometimes even stronger, than each of the monometallic surfaces.

It is apparent that bimetallic surfaces can have markedly different reactivity than monometallic surfaces, and an appropriate treatment of bimetallic catalysts will require the analysis of bimetallic alloyed systems. Since an exhaustive treatment of adsorbate interaction with all possible bimetallic ensembles is practically impossible, there is considerable value in determining the important electronic parameters of the surface that control surface reactivity and evaluating how bimetallic interactions affect them.

Table II summarizes the electronic properties of the bare surfaces of monometallic $\mathrm{Pd}(111), \operatorname{Re}(0001)$ and the alloyed $\mathrm{Pd}-\mathrm{Re}$ surfaces. The $d$-band filling for the surface metal atoms, reported in Table II, were determined by calculating the fractional area under the curve of the density-of-states (DOS) projected to the $d$ band, which is below the Fermi energy. The $d$-band center for the surface metal atoms were calculated by taking the first moment of the normalized projected DOS about the Fermi level. From Table II, it appears that bimetallic $\mathrm{Pd}-\mathrm{Re}$ interaction in the alloyed surfaces has nominal effect on the $d$-band filling for Pd and Re. The Pd $d$-band filling is $0.95-0.96$ and the Re $d$-band filling is between 0.68 and 0.73 , in all cases. It therefore appears that the significant changes in the hydrogen adsorption energy cannot be attributed to charge-transfer effects alone. In Fig. 2, we have plotted the projected DOS for the alloyed Pd-Re overlayers and uniformly alloyed surfaces. The solid lines depict the DOS projected to the surface $\operatorname{Pd} d$ band. The dotted lines
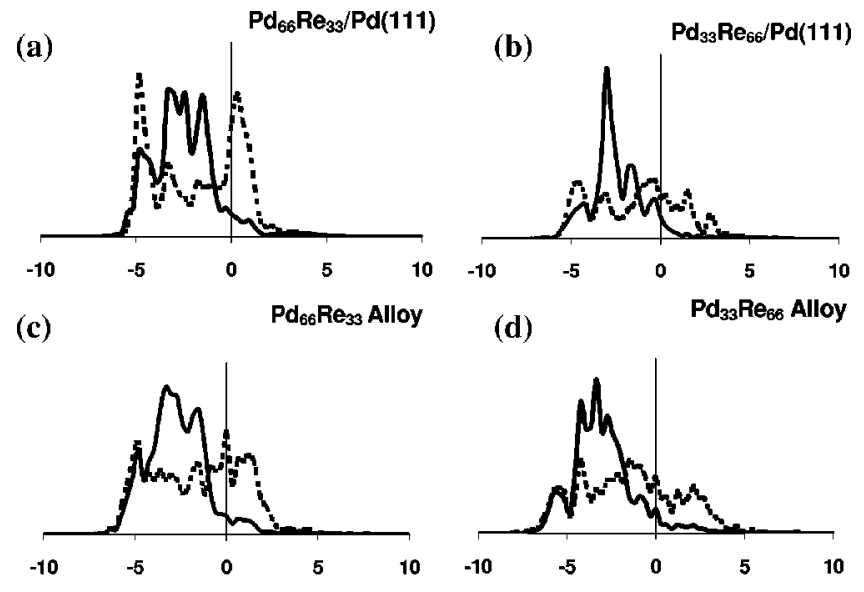

(e)
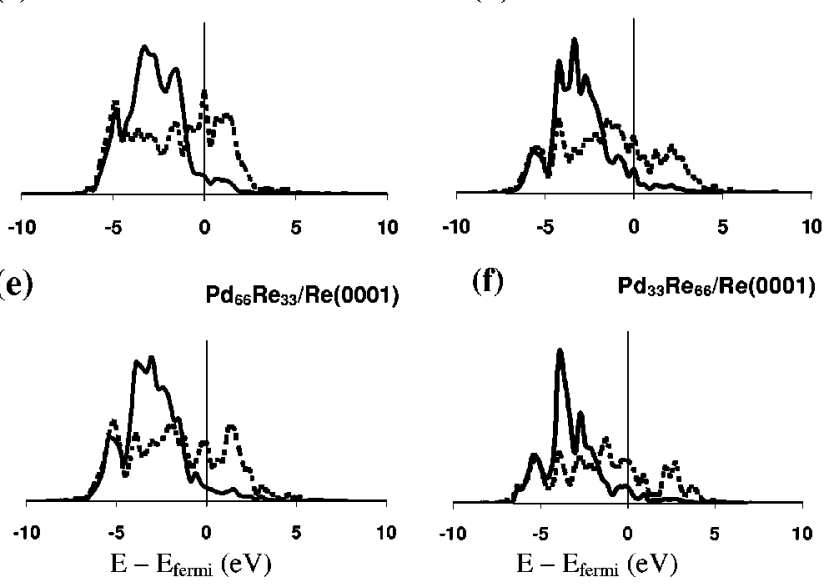

FIG. 2. DFT-GGA computed electronic density of states (DOS) for Pd-Re alloyed overlayers and surfaces. Solid lines correspond to DOS projected to the $d$ band for the surface Pd atoms. Dotted lines correspond to the DOS projected to the $d$ band of the surface Re atoms. 
correspond to the projection onto the $d$ band for the surface Re atoms. It is evident that there are significant differences in the shape of the $d$ band (see Fig. 2) due to bimetallic alloying. Integration under the curve up to the Fermi level indicates that the $d$-band filling remains approximately the same. The changes in the shape of the $d$-band primarily affect the location of the $d$-band center, i.e., the first moment of the normalized projected DOS. This effect is more predominant in the Pd $d$-band structure as compared to that for Re. In examining Fig. 2, the plots from top to bottom [(a)-to-(c)-to(e) and (b)-to-(d)-to-(f)], show the effect on the surface metal $d$ band due to increasing the amount of Re in the substrate. It appears that increasing the amount of $\mathrm{Re}$ in the substrate shifts the weight of the Pd $d$ band away from the Fermi energy. Looking from left-to-right in Fig. 2 [(a) and (b); (e) and (f)], we see the effect of increasing the amount of Re in the surface layer. The $d$ band for the surface Pd atoms below the Fermi level is observed to shift further away from the Fermi energy as the number of Re neighbors is increased. From Table II, the $d$ band center for Pd is observed to vary anywhere between $-0.44 \mathrm{eV}$ and $-1.18 \mathrm{eV}$ from the $d$-band center for $\operatorname{Pd}(111)(-1.98 \mathrm{eV})$. The changes in the $\operatorname{Re} d$ band due to the presence of Pd neighbors, on the surface and in the bulk, are relatively subtle. There are smaller shifts in the $d$-band position due to bimetallic $\mathrm{Pd}-\mathrm{Re}$ interaction. The $\mathrm{Re}$ $d$-band center for the bimetallic surfaces, which are tabulated in Table II, vary between $-0.18 \mathrm{eV}$ and $-0.53 \mathrm{eV}$ relative to $\operatorname{Re}(0001)(-1.16 \mathrm{eV})$. Oelhafen and co-workers have experimentally demonstrated similar shifts in the valence $d$-band of metals for amorphous alloys such as $\mathrm{Pd}-\mathrm{Zr}, \mathrm{Cu}-\mathrm{Zr}$, and $\mathrm{Au}-\mathrm{Sn}$, using ultraviolet photoelectron spectroscopy. ${ }^{38,39}$ They find that increasing the $\mathrm{Zr}$ content in a $\mathrm{Pd}-\mathrm{Zr}$ alloy shifts the average Pd $d$-band position to higher binding energies by as much as $2 \mathrm{eV}$. The shift in the $\mathrm{Zr} d$ band is relatively small due to forming the bimetallic amorphous alloy. Our results seem to be consistent with the experimental observation. $^{38,39}$

In Ref. 21, a simple model was proposed that correlated changes in the metal-adsorbate bond energy to changes in the metal $d$-band center for the bare-metal surface. In a previous paper, we demonstrated that the hydrogen binding energies on monometallic $\operatorname{Pd}(111), \operatorname{Re}(0001)$, and pseudomorphic $\mathrm{Pd}_{\mathrm{ML}} / \operatorname{Re}(0001)$ and $\mathrm{Re}_{\mathrm{ML}} / \mathrm{Pd}(111)$ overlayers correlate with the $d$-band center for the surface metal atom. ${ }^{25}$ In this paper, we extend the model to correlate the hydrogen chemisorption energy on alloyed $\mathrm{Pd}-\mathrm{Re}$ overlayers and surfaces by using a weighted $d$-band center as the key parameter to represent the surface electronic structure.

For adsorption of hydrogen on an alloyed Pd-Re surface, the $d$-band positions for the Pd and Re atoms on the surface are markedly different. The extent of overlap between hydrogen and the $d$ bands of Pd and Re are also different. This is seen as a significantly elongated $\mathrm{Pd}-\mathrm{H}$ and contracted $\mathrm{Re}-\mathrm{H}$ bond distances on the alloyed $\mathrm{Pd}-\mathrm{Re}$ surfaces. It is therefore necessary to use an appropriate weighting scheme to account for the differences in the interaction of $\mathrm{Pd}$ and Re with hydrogen. Since the coupling of the hydrogen $1 s$ state with the metal $d$ band is proportional to the $d$-band coupling matrix element, it seems to be an appropri-

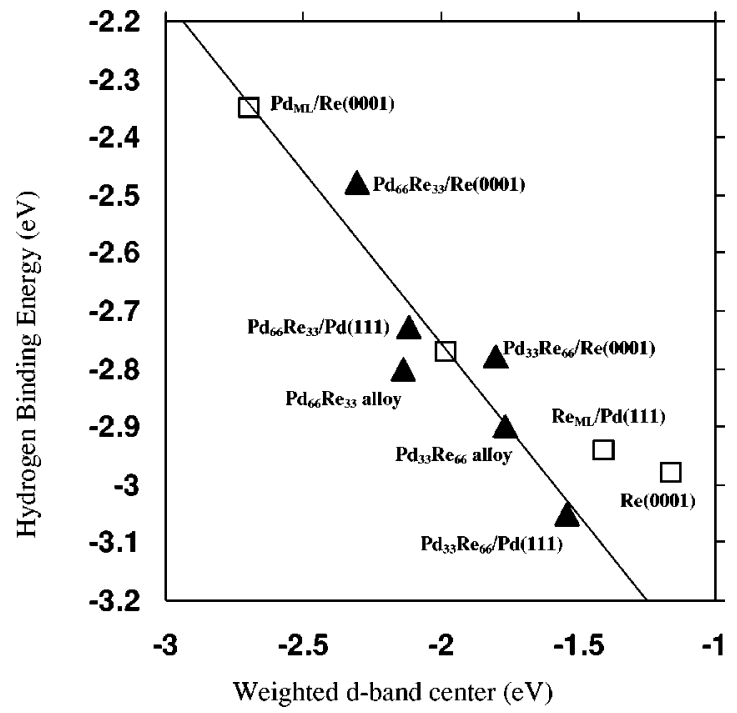

FIG. 3. DFT-GGA hydrogen chemisorption energy on $\operatorname{Pd}(111), \operatorname{Re}(0001)$ and bimetallic Pd-Re surfaces plotted as a function of the weighted $d$-band center for the surface metal atoms. The filled triangles correspond to the bimetallic surface where the $d$-band weighting scheme of Eq. (1) was used. The open squares correspond to surfaces where hydrogen is bound to a single type of metal atom.

ate weighting factor to calculate the weighted $d$-band center. The weighted $d$-band centers for the surface were therefore determined using Eq. (1):

$$
\epsilon_{d \text {-weighted }}=\frac{\left(V_{\mathrm{Re}}^{2} \cdot \epsilon_{d}^{\mathrm{Re}} \cdot N^{\mathrm{Re}}+V_{\mathrm{Pd}}^{2} \cdot \epsilon_{d}^{\mathrm{Pd}} \cdot N^{\mathrm{Pd}}\right)}{\left(V_{\mathrm{Re}}^{2} \cdot N^{\mathrm{Re}}+V_{\mathrm{Pd}}^{2} \cdot N^{\mathrm{Pd}}\right)},
$$

where, $\epsilon_{d \text {-weighted }}$ is the weighted $d$-band center for the surface, used to assess the surface reactivity using the model of Ref. 21. $V^{2}$ is the $d$-band coupling matrix element for the surface metal atom $\left[V_{\mathrm{Pd}}^{2}=2.78\right.$ and $\left.\left.V_{\mathrm{Re}}^{2}=6.04\right]\right]^{2,21} N^{\mathrm{Re}}$ and $N^{\mathrm{Pd}}$ are the number of $\mathrm{Re}-\mathrm{H}$ and $\mathrm{Pd}-\mathrm{H}$ bonds, respectively, formed by the chemisorbed hydrogen atom. The $d$-band centers calculated using this weighting scheme are tabulated in Table II. In Ref. 25, we showed that both Pd and Re surfaces can be displayed on the same graph for analysis using the model of Ref. 21. In Fig. 3, we have plotted the hydrogen chemisorption energy as a function of the weighted $d$-band center for the different alloyed Pd-Re overlayers and surfaces. It appears that there is a strong correlation between the $d$-band centers for the surface metal atoms and the hydrogen chemisorption energies. The farther the weighted d-band center is shifted below the Fermi energy, the weaker is the interaction of atomic hydrogen with the surface metal layer. This correlation is also valid for the alloyed systems where hydrogen is bound to both $\mathrm{Pd}$ and Re concurrently.

\section{CONCLUSIONS}

To summarize, we have analyzed the adsorption of atomic hydrogen in the three-fold fcc site on various alloyed $\mathrm{Pd}-\mathrm{Re}$ surfaces. The calculations indicate a large variation $(0.7 \mathrm{eV})$ in the $\mathrm{BE}$ of atomic hydrogen due to bimetallic alloying. These changes cannot be accounted by charge transfer effects alone in the bimetallic surface. We have extended the original model of Ref. 21 to the analysis of hy- 
drogen chemisorption on alloyed Pd-Re surfaces. It is demonstrated that the $d$-band center, weighted by the $d$-band coupling matrix element, provides an appropriate parameter by which to correlate the surface reactivity of bimetallic $\mathrm{Pd}-\mathrm{Re}$ surfaces. Finally, in analyzing the $d$ bands for the bare Pd-Re surfaces, it is observed that increasing the number of Re neighbors around Pd shifts the $d$-band center for Pd away from the Fermi energy. The same is true for the surface Re atoms, but the shift in the $d$-band center due to increasing the number of Pd neighbors is relatively small.

\section{ACKNOWLEDGMENTS}

We would like to thank Bjørk Hammer, Manos Mavrikakis, and Andrei Ruban for helpful discussions. The present work was in part financed by The Danish Research Councils through the Center for Surface Reactivity and Grants Nos. 9501775 and 9800425 . The Center for Atomic Scale Materials Physics is sponsored by the Danish National Research Foundation. Acknowledgment is also made to the donors of the Petroleum Research Fund (Grant No. 31342G5), administered by the ACS, for support of this work. The DuPont Chemical Company (USA) and NSF (Award No. CTS-9702762) are also acknowledged for financial support.

${ }^{1}$ J. H. Sinfelt, Bimetallic Catalysts: Discoveries, Concepts and Applications (Wiley, New York, 1983).

${ }^{2}$ A. Ruban et al., J. Mol. Catal. A: Chem. 115, 421 (1997).

${ }^{3}$ B. Hammer, Y. Morikawa, and J. K. Nørskov, Phys. Rev. Lett. 76, 2141 (1996).

${ }^{4}$ M. Ø. Pedersen et al., Surf. Sci. 426, 395 (1999).

${ }^{5}$ V. Pallassana, M. Neurock, and G. W. Coulston, J. Phys. Chem. B 103, 8973 (1999).

${ }^{6}$ J. A. Rodriguez and D. W. Goodman, J. Phys. Chem. 95, 4196 (1991).

${ }^{7}$ J. A. Rodriguez and D. W. Goodman, Science 257, 897 (1992).
${ }^{8}$ B. Frühberger, J. Eng, Jr., and J. G. Chen, Catal. Lett. 45, 85 (1997).

${ }^{9}$ J. W. He, W. L. Shea, X. Jiang, and D. W. Goodman, J. Vac. Sci. Technol. A 8, 2435 (1990).

${ }^{10}$ R. M. Ormerod, C. J. Baddeley, and R. M. Lambert, Surf. Sci. Lett. 259, L709 (1991).

${ }^{11}$ B. E. Koel, A. Sellidj, and M. T. Paffett, Phys. Rev. B 46, 7846 (1992).

${ }^{12}$ J. H. Larsen and I. Chorkendorff, Surf. Sci. 405, 62 (1998).

${ }^{13}$ C. Xu and W. D. Goodman, Langmuir 12, 1807 (1996).

${ }^{14}$ J. A. Rodriguez, Surf. Sci. 345, 347 (1996).

${ }^{15}$ R. A. Campbell, J. A. Rodriguez, and D. W. Goodman, Phys. Rev. B 46, 7077 (1992).

${ }^{16} \mathrm{R}$. Hoffmann, Solids and Surfaces, A Chemist's View of Bonding in Extended Surfaces (VCH, New York, 1988).

${ }^{17}$ R. A. van Santen, Theoretical Heterogeneous Catalysis (World Scientific, Singapore, 1991).

${ }^{18}$ D. M. Newns, Phys. Rev. B 178, 1123 (1969).

${ }^{19}$ B. Hammer and J. K. Nørskov, in Chemisorption and Reactivity on Supported Clusters and Thin Films, edited by R. M. Lambert and G. Pacchioni (Kluwer Academic, Dordrecht, The Netherlands, 1997), pp. 285351.

${ }^{20}$ B. Hammer and J. K. Nørskov, Nature (London) 376, 238 (1995).

${ }^{21}$ B. Hammer and J. K. Nørskov, Surf. Sci. 343, 211 (1995).

${ }^{22}$ B. Hammer, O. H. Nielsen, and J. K. Nørskov, Catal. Lett. 46, 31 (1997).

${ }^{23}$ A. Eichler, G. Kresse, and J. Hafner, Surf. Sci. 397, 116 (1998).

${ }^{24}$ D. M. Bird, Faraday Discuss. Chem. Soc. 110, 335 (1998).

${ }^{25}$ V. Pallassana et al., Phys. Rev. B 60, 6146 (1999).

${ }^{26}$ M. Mavrikakis, B. Hammer, and J. K. Nørskov, Phys. Rev. Lett. 81, 2819 (1998).

${ }^{27}$ B. Hammer and J. K. Nørskov, Phys. Rev. Lett. 79, 4441 (1997).

${ }^{28}$ V. Pallassana and M. Neurock, J. Catal. (in press).

${ }^{29}$ J. P. Perdew et al., Phys. Rev. B 46, 6671 (1992).

${ }^{30}$ S. J. Vosko, L. Wilk, and M. Nusair, Can. J. Phys. 58, 1200 (1980).

${ }^{31}$ M. C. Payne et al., Rev. Mod. Phys. 64, 1045 (1992).

${ }^{32}$ N. Troullier and J. L. Martins, Phys. Rev. B 43, 1993 (1991).

${ }^{33}$ D. J. Chadi and M. L. Cohen, Phys. Rev. B 8, 5747 (1973).

${ }^{34}$ J. F. Paul and P. Sautet, Phys. Rev. B 53, 8015 (1996).

${ }^{35}$ J. F. Paul and P. Sautet, Surf. Sci. 356, L403 (1996).

${ }^{36}$ H. Conrad, G. Ertl, and E. E. Latta, Surf. Sci. 41, 435 (1974).

${ }^{37}$ D. J. Godbey and G. A. Somorjai, Surf. Sci. 204, 301 (1988).

${ }^{38}$ P. Oelhafen, E. Hauser, H.-J. Guntherodt, and K. H. Bennemann, Phys. Rev. Lett. 43, 1134 (1979).

${ }^{39}$ P. Haussler et al., Phys. Rev. Lett. 51, 714 (1983). 